\title{
Vorstandswahlen der Arbeitsgemeinschaften der DRG auf dem 98. Deutschen Röntgenkongress und 8. Gemeinsamen Kongress der DRG und ÖRG, 24.-27. Mai 2017 in Leipzig
}

Auf dem diesjährigen Röntgenkongress finden für folgende Arbeitsgemeinschaften Vorstandswahlen statt:

- AG Diagnostische Radiologie arbeitsund umweltbedingter Erkrankungen

- AG Kopf/Halsdiagnostik

- AG Bildgebende Verfahren des Bewegungsapparates

- AG Ultraschall

- AG Onkologische Bildgebung

- AG Pädiatrische Radiologie

- AG Uroradiologie/Urogenitaldiagnostik

Entsprechend der Arbeitsordnung der Arbeitsgemeinschaften bedürfen die zur Wahl vorgeschlagenen Kandidaten der Zustimmung des Vorstandes der DRG (nihil obstat). Die Mitglieder der oben genannten Arbeitsgemeinschaften werden hiermit eingeladen, Kandidaten für den Vorstand der Wahlperiode 2017 - 2019 vorzuschlagen. Die Vorschläge müssen schriftlich eingereicht werden und sollten das Einverständnis der vorgeschlagenen Kandidaten enthalten.

\section{AG Diagnostische Radio-} logie arbeits- und umweltbedingter Erkrankungen

Der Vorstand der AG Diagnostische Radiologie arbeits- und umweltbedingter Erkrankungen bittet seine Mitglieder um Kandidatenvorschläge für die Vorstandswahl 2017. Als Kandidaten können nur Mitglieder vorgeschlagen werden, die am 1. März 2017 ordentliche Mitglieder der Arbeitsgemeinschaft waren. Vorschläge bedürfen der Schriftform und sind bis spätestens 12. März 2017 an den Vorstand der AG Diagnostische Radiologie arbeits- und umweltbedingter Erkrankungen zu Händen der Vorsitzenden, Dr. Renate Rehbock, Berlin, oder an die Geschäftsstelle der DRG z. Hd. Frau Engelhardt zu senden.

\section{AG Kopf/Halsdiagnostik}

Der Vorstand der AG Kopf/Halsdiagnostik bittet seine Mitglieder um Kandidatenvorschläge für die Vorstandswahl 2017. Als
Kandidaten können nur Mitglieder vorgeschlagen werden, die am 1. März 2017 ordentliche Mitglieder der Arbeitsgemeinschaft waren. Vorschläge bedürfen der Schriftform und sind bis spätestens 12. März 2017 an den Vorstand der Arbeitsgemeinschaft zu Händen des Vorsitzenden, Prof. Dr. Florian Dammann, Bern, oder an die Geschäftsstelle der DRG z. Hd. Frau Minkova zu senden.

\section{AG Bildgebende Verfahren des Bewegungsapparates}

Der Vorstand der AG Bildgebende Verfahren des Bewegungsapparates bittet seine
Mitglieder um Kandidatenvorschläge für die Vorstandswahl 2017. Als Kandidaten können nur Mitglieder vorgeschlagen werden, die am 1. März 2017 ordentliche Mitglieder der Arbeitsgemeinschaft waren. Vorschläge bedürfen der Schriftform und sind bis spätestens 12. März 2017 an den Vorstand der Arbeitsgemeinschaft zu Händen des Vorsitzenden Prof. Dr. KarlFriedrich Kreitner, Mainz, oder an die Geschäftsstelle der DRG z. Hd. Frau Minkova zu senden.

\section{AG Ultraschall}

Der Vorstand der AG Ultraschall bittet seine Mitglieder um Kandidatenvorschläge für 
die Vorstandswahl 2017. Als Kandidaten können nur Mitglieder vorgeschlagen werden, die am 1. März 2017 ordentliche Mitglieder der Arbeitsgemeinschaft waren. Vorschläge bedürfen der Schriftform und sind bis spätestens 12. März 2017 an den Vorstand der Arbeitsgemeinschaft zu Händen des Vorsitzenden Prof. Dr. Ulf Teichgräber, Jena, oder an die Geschäftsstelle der DRG z. Hd. Frau Engelhardt zu senden.

\section{AG Onkologische Bildgebung}

Der Vorstand der AG Onkologische Bildgebung bittet seine Mitglieder um Kandidatenvorschläge für die Vorstandswahl 2017. Als Kandidaten können nur Mitglieder vorgeschlagen werden, die am 1. März 2017 ordentliche Mitglieder der Arbeitsgemeinschaft waren. Vorschläge bedürfen der Schriftform und sind bis spätestens 12. März 2017 an den Vorstand der Arbeitsgemeinschaft zu Händen des Vorsitzenden Prof. Dr. Heinz Schlemmer, Tübingen, oder an die Geschäftsstelle der DRG z. Hd. Frau Ermisch zu senden.

\section{AG Pädiatrische Bildgebung}

Der Vorstand der AG Pädiatrische Bildgebung bittet seine Mitglieder um Kandidatenvorschläge für die Vorstandswahl 2017. Als Kandidaten können nur Mitglieder vorgeschlagen werden, die am 1. März 2017 ordentliche Mitglieder der Arbeitsgemeinschaft waren. Vorschläge bedürfen der Schriftform und sind bis spätestens 12. März 2017 an den Vorstand der Arbeitsgemeinschaft zu Händen des Vorsitzenden
PD Dr. Christoph Heyer, Bochum, oder an die Geschäftsstelle der DRG z. Hd. Frau Schlender zu senden.

\section{AG Uroradiologie/Urogeni- taldiagnostik}

Der Vorstand der AG Uroradiologie/Urogenitaldiagnostik bittet seine Mitglieder um Kandidatenvorschläge für die Vorstandswahl 2017. Als Kandidaten können nur Mitglieder vorgeschlagen werden, die am 1. März 2017 ordentliche Mitglieder der Arbeitsgemeinschaft waren. Vorschläge bedürfen der Schriftform und sind bis spätestens 12. März 2017 an den Vorstand der Arbeitsgemeinschaft zu Händen des Vorsitzenden Dr. Matthias Röthke, Hamburg, oder an die Geschäftsstelle der DRG z. Hd. Frau Bruchalska zu senden. 